\title{
Real-Time Air-to-Ground Data Communication Technology of Aeroengine Health Management System with Adaptive Rate in the Whole Airspace
}

\author{
Qiuying Yan,, ${ }^{1}$ Wei Li $\mathbb{D}^{1}{ }^{1}$ Jiacheng Li, ${ }^{2}$ Jie Zhang, ${ }^{2}$ Shengyi Liu, ${ }^{2}$ Zhe Wang, ${ }^{2}$ Tong Liu, ${ }^{2}$ \\ Qian Chen, ${ }^{2}$ and Hanlin Sheng $\mathbb{D}^{2}$ \\ ${ }^{1}$ AECC Hunan Aviation Powerplant Research Institute, Zhuzhou, Hunan 412002, China \\ ${ }^{2}$ Nanjing University of Aeronautics and Astronautics, Nanjing, Jiangsu 210016, China \\ Correspondence should be addressed to Wei Li; buaa608@sina.com and Hanlin Sheng; dreamshl@qq.com
}

Received 22 March 2021; Accepted 26 June 2021; Published 31 July 2021

Academic Editor: Zain Anwar Ali

Copyright (C) 2021 Qiuying Yan et al. This is an open access article distributed under the Creative Commons Attribution License, which permits unrestricted use, distribution, and reproduction in any medium, provided the original work is properly cited.

\begin{abstract}
To overcome the problem of data transmission of the aeroengine health management system, a multilink communication system combining ultrahigh-frequency communication link, $4 \mathrm{G}$ cellular mobile communication link, and BeiDou satellite communication link was proposed. This system can realize the functions such as data receiving and sending, data encryption, and resuming transfer from the break point based on multiple links. When the flight altitude is not high, the communication distance is short, so the UHF digital transmission radio communication link is adopted, which is highly efficient and stable. When the communication distance is long, the $4 \mathrm{G}$ cellular mobile communication link can ensure both the communication distance and the communication rate. In the area where $4 \mathrm{G}$ signal cannot be covered in extreme terrain environment, BeiDou satellite communication link is used for data transmission. Besides, in order to ensure the communication rate of the link, a multilink adaptive switching technology was also developed. The test verified that the system can perform adaptive switching among multiple links, realize air-ground data communication in the whole airspace, and achieve a good communication rate, which has significative value of engineering application.
\end{abstract}

\section{Introduction}

With the gradual expansion of domestic aviation platforms and the rapid growth of the transportation industry, the requirements for real-time, safe, and reliable air-to-ground communication technology have continuously improved. The air-to-ground data link system for engine health management, as the main method of real-time monitoring of engine status, has been extensively applied in civil aviation owing to its fast transmission, strong anti-interference ability, low bit error rate, and high reliability $[1,2]$.

The failure strategy of engine gas circuit performance monitoring, fault diagnosis, and response is an important part of engine health management $[3,4]$. In the aviation industry, flight accidents are often caused by engine failures. Therefore, it is extremely important to strengthen real-time monitoring of engine operating status. Although health management technology is the most effective method to ensure the safe operation of the aeroengine and reduce maintenance costs, the timeliness and accuracy of data sources used by it will directly affect the quality of health assessment. In terms of algorithm theory research on aeroengine health management, typical algorithms include methods based on physical models $[5,6]$, methods based on data $[7,8]$, methods based on experience, and fusion methods based on intelligent algorithms [9-11].

During the flight, the air-to-ground data link system realizes real-time monitoring of engine operating status by continuously monitoring the operating status of the aircraft, sending the on-board information to the ground terminal in real time, and using the powerful ground network control processing center to analyze and process it, so as to provide 
constructive decision-making reference for crew members, facilitate the preparation for maintenance before the aircraft landing, shorten maintenance time, and guarantee efficient engine operation $[12,13]$. The acquisition of potential engine failure information is the prerequisite for enhancing maintenance efficiency, saving costs, and guaranteeing flight safety. Therefore, it is particularly essential to study the communication technology of air-to-ground data on health management of aeroengine $[14,15]$.

In the 1940s, the communication in civil aviation mainly relied on the very high-frequency (VHF) communication system [16], which was gradually used as a global aviation mobile service; thus, the VHF communication in aviation has been fully realized $[17,18]$. However, in recent years, with the gradual opening of low-altitude airspace, it has become a hot trend to use aerial vehicle for low-altitude flight. Restricted by the flying height and small speed of aircraft, high-frequency aviation communication is difficult to be effectively covered at low altitude, leading to insufficient communication safety guarantee for low-altitude flight. The $4 \mathrm{G}$ cellular mobile communication system is suitable for small low-flying aerial vehicles with small flight speed; coupled with a large number of existing base stations and the low construction cost, it can provide a certain degree of communication guarantee $[19,20]$. However, mobile base stations cannot be fully covered in remote areas such as the sea, deserts, mountainous areas, and forests, which restricts the development of network communication to a large extent. Therefore, the satellite communication system with long communication distance, wide working frequency, stable and reliable communication, and high communication quality has been widely applied in the field of aviation communication $[21,22]$.

To meet the requirements for air-to-ground data communication of the aeroengine health management system in the whole airspace, a communication technology that integrates three air-to-ground data links was proposed in this paper, which can realize communication in different flight scenarios. Besides, based on the respective advantages of the three data links, a multilink adaptive switching technology was developed to ensure the quality of air-to-ground data communication. In order to ensure the integrity and security of the data in the process of data transfer, the technologies of resuming transfer from the break point and data encryption were used, which enhanced the accuracy and reliability of the whole airspace air-to-ground data communication of the aeroengine health management system.

In this paper, the first part mainly introduces the realtime communication scheme of air-to-ground data on health management and studies related key technologies to lay a good foundation for the research path of system design, mainly including air-ground segment, and ground-ground segment real-time communication technology, key information encryption technology, key information resuming transfer from the break point technology, and the technology of air-to-ground data communication with adaptative rate. The second part mainly describes the construction of a prototype system for real-time communication of air-toground data of health management, which mainly includes the construction of a two-way transmission architecture for air-ground segment, ground-ground segment data, and the implementation of each component of the prototype system for real-time air-to-ground data communication. The third part mainly presents a simulated flight test on real-time communication and transmission of air-to-ground data of health management and verifies the communication of these three communication links and the adaptive switching technology of the system. It was confirmed that the system can realize the adaptive communication among the three links and achieve a good communication rate.

\section{The Scheme of Real-Time Air-to-Ground Data Communication of Health Management}

2.1. Research on Real-Time Air-Ground Segment, GroundGround Segment, and Relaying Communication Technologies. The health management system of aeroengine mainly monitors the operating status of aircraft engine and sends relevant health management data in real time to the ground control center, which, after processing and analysis, distributes it to relevant departments to take corresponding measures [23]. This system involves the communication of two processes, namely, the air-ground segment and groundground segment. Therefore, the scheme of air-to-ground data communication of health management requires separate research on these two processes.

\subsubsection{Research on Air-Ground Segment Communication} Technology. At present, China has formed an air-to-ground communication system dominated by VHF data communication and assisted by satellite communication. In this context, the air-ground segment communication of the health management system for aeroengine needs to meet the requirements for communication in different flight airspace. First, within a limited distance, the communication of the ultrahighfrequency (UHF) digital radio station link is the safest and fastest. Characterized by fast data update and small communication delay, it can provide reliable air-to-ground communication. Under nonhorizon communication, the $4 \mathrm{G}$ cellular mobile communication link is significantly superior to the communication of UHF digital radio station, with the advantages of mature technology, high rate, and small delay. However, 4G communication is restricted in remote areas where base stations have weak air-to-air signals or where it is difficult to deploy base stations. In such case, the BeiDou satellite communication link can give play to its own advantages. The short message function, which is the unique communication function of the BeiDou satellite, can achieve reliable communication in remote areas such as the sea, the fields, and the mountainous areas thanks to its advantages, such as wide coverage and strong anti-interference ability [24].

Considering the applicable scenarios and transmission range of different links, a combination of three data links, UHF data transfer communication, 4G cellular mobile communication, and BeiDou satellite communication, was adopted in the system, so as to make full use of the advantages of the three communication links to ensure the 
communication of the entire communication system in the whole airspace. Figure 1 shows the air-ground segment data link of the health management system.

\subsubsection{Research on Ground-Ground Segment Communication} Technology. During the health data management of the aeroengine, the ground control center needs to process and transmit the obtained health data to multiple departments. To realize the transmission of data to multiple departments, ground-ground segment communication needs to meet the requirements for one-to-many or many-to-many interactive communication, so as to realize data sharing.

To allow the health management data to be accurately and timely distributed to other departments and to realize the sharing of engine's health management data, after research and tests, the ground-ground segment adopted the user datagram protocol (UDP) network communication protocol for communication.

UDP network communication protocol is a connectionless transport layer protocol, with the advantages such as small resource consumption, fast processing, and high real-time performance. In addition, it supports one-tomany and many-to-many interactive communication, which can achieve the function of transmitting health management data to different departments. Figure 2 exhibits the groundground segment data link of the health management system.

2.2. Research on the Technology of Compression and Encryption of Key Health Management Information. As the "heart" of the aircraft, the aeroengine represents the highest level of industrial technology of a country and is hailed as the "Pearl" on the "Crown" of industry. Thus, its health management data are evidently vital. In the communication system for air-to-ground data of health management, the data need to be processed to prevent it from being stolen, attacked, and destroyed. Before transmitted, the data can be encrypted to reduce threats and ensure data security to the greatest extent.

The security encryption algorithms for information transmission have been extensively studied, and in this system, DES security encryption algorithm was used. DES is a world-recognized standard encryption format, which performs encryption by 64-bit data packets before replacement and iteration $[25,26]$. The length of the key is 56 bits, and processing such as replacement and cyclic shift is also required. In the initial IP replacement, the 64-bit plaintext is disorganized according to an $8 * 8$ replacement table to increase the strength of encryption; subkey is used in 16 iterative computations; the initial ciphertext formed by each packet undergoes the inverse initial replacement again, which is the inverse operation of the initial replacement, to obtain the final ciphertext of the packets. The specific process is displayed in Figure 3.

2.3. Research on the Technology of Resuming Transfer from the Break Point of Key Health Management Information. Resuming transfer from the break point means that, during the data upload or download process, when the connection is reestablished after an interruption, the part that has never been uploaded or downloaded will continue to be transferred without repeating the transmission of the existing content. This technology can effectively save time and improve the efficiency of the system $[27,28]$.

There are two key issues that need to be paid attention to in resuming transfer from the break point:

(1) When the transmission is interrupted, the local download information is saved: a temporary file needs to be created to save the local download information, including the number of downloaded bytes, the file pointer, and the total file size, so that the download information will be stored in a temporary file when the transmission is interrupted, and the file pointer will point to the end of the downloaded file.

(2) The local download information needs to be checked when resuming the transfer from the break point: first, it is necessary to determine whether the downloaded file exists locally; if it exists, the download information of the downloaded file is read, and the pointer is moved to the next position of the downloaded bytes at the same time so that the transfer of the file can be resumed from the break point.

Figure 4 shows the flowchart of resuming transfer from the break point.

\subsection{Research on the Technology of Air-to-Ground Data} Communication of Health Management with Adaptive Rate. Different communication links are applicable to different working scenarios, working ranges, and transmission distances. If only one communication link is used for communication, the communication effect will vary among different scenarios where the aircraft performs tasks. The application of multiple communication links can not only take advantage of each communication link to ensure communication quality but also improve the survivability of the entire communication system. Therefore, it is necessary to use multiple data links for communication.

When multiple communication links exist in the system, how to coordinate these communication links to allow them to work efficiently is a key issue that needs to be solved. Therefore, an adaptive switching mechanism based on three communication links was investigated. Considering the differences in the transmission range and update frequency among UHF digital radio station, 4G cellular mobile communication, and BeiDou satellite communication, the default priority of the communication links (from high to low) was set as follows: UHF digital radio station, $4 \mathrm{G}$ cellular mobile communication, and BeiDou satellite communication. Even if the information of several communication links is received simultaneously, the ground control center will only process and distribute the data of the communication link with the highest priority currently. This mechanism conducts analysis according to the packet loss and transmission delay to judge the communication quality of the 


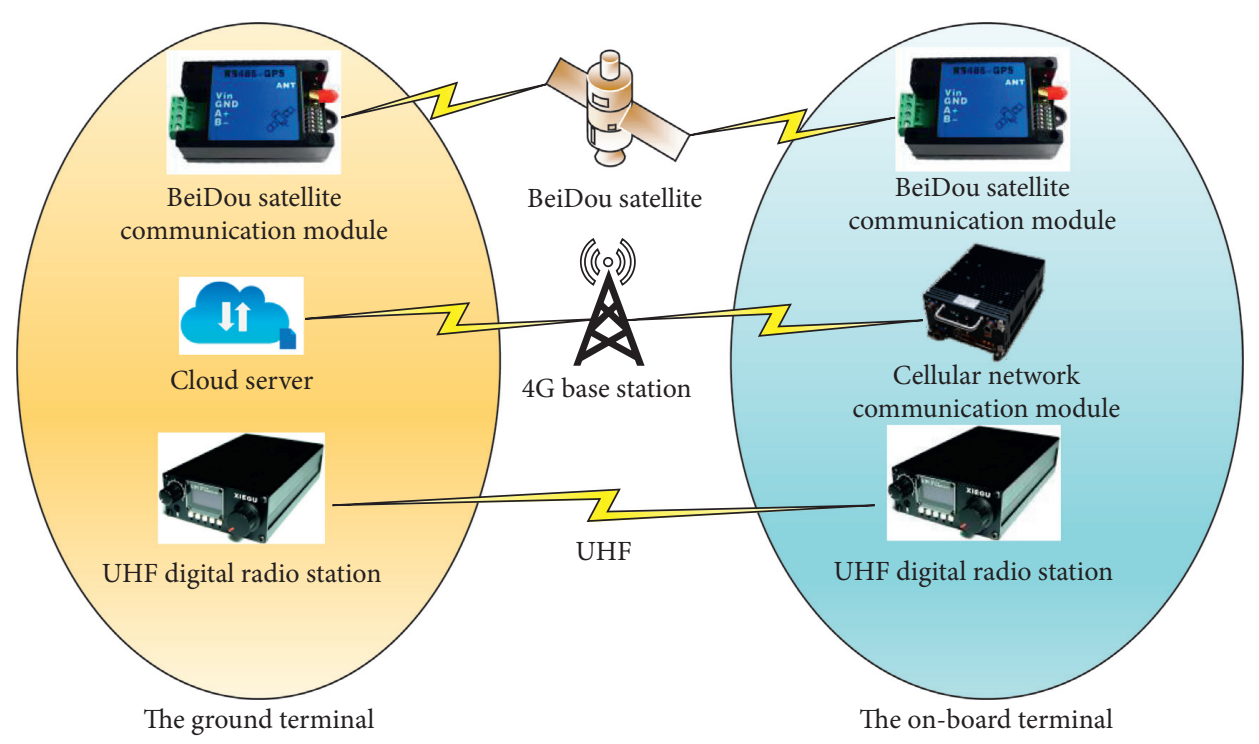

FIGURE 1: Diagram of air-ground segment data link.

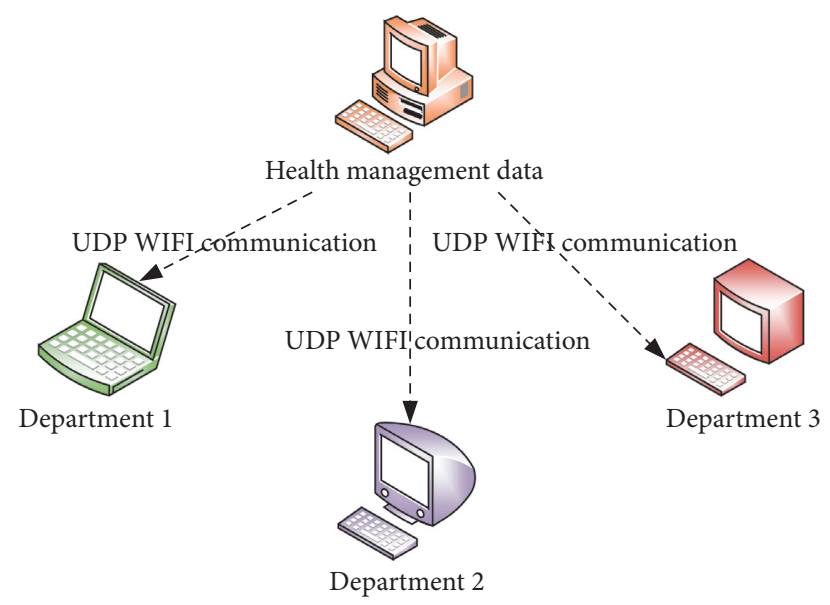

Figure 2: Diagram of the ground-ground segment data link.

existing communication link. If the communication quality deteriorates and the optimal data cannot be provided, it will switch to other communication links for communication according to the priority. For example, when the transmission delay is greater than $500 \mathrm{~ms}$ or the packet loss rate is greater than $1 \%$, the communication condition is considered bad, and the communication link needs to be switched, as shown in Figure 5.

\section{Construction of a Prototype System for Real- Time Communication of Air-to-Ground Health Management Data}

3.1. Construction of a Two-Way Data Transfer Architecture for Air-Ground Segment and Ground-Ground Segment Data. The main cross-linking relationship in the prototype system for real-time communication of air-to-ground data of health management is displayed in Figure 6. The on-board flight control system transmits data with the engine simulation equipment through serial communication, and the engine simulation equipment and the on-board terminal of data link realize data interaction through serial communication. The ground terminal of the data link realizes information interaction with the ground control center through the serial port, and the ground control center conducts information interaction with the outfield portable maintenance aids (PMA), the simulation equipment of the maintenance center, and the support center through the network. The on-board terminal and the ground terminal of data link communicate through three links: UHF digital radio station, $4 \mathrm{G}$ cellular network communication module, and BeiDou satellite communication module, and switching is performed among the three communication methods through an adaptive switching mechanism to select an optimal communication method, so as to ensure reliable and stable communication.

The flight control system sends data such as flight status information to the engine simulation equipment, which calculates the telemetry data of the simulated engine according to the received parameters such as the amount of the throttle lever of the flight control system, and transmits it to the on-board terminal of data link. The data link on-board terminal then frames the received telemetry data from the flight control system and the engine, as well as the status data from the on-board terminal of data link, and then transmits the data to the data link ground terminal through the cellular network communication module, BeiDou satellite communication, and UHF digital radio station after the data are encrypted, encoded, and modulated. The ground RF front end of the data link ground terminal converts the received radio frequency signal before transmitting it to the ground terminal of data link, which transfers the data to the ground control center after processing such as demodulation, decoding, and decryption. The ground control center analyzes and reframes the received data and then distributes it to the simulation equipment of the outfield PMA terminal, maintenance center, and support center through network communication. 


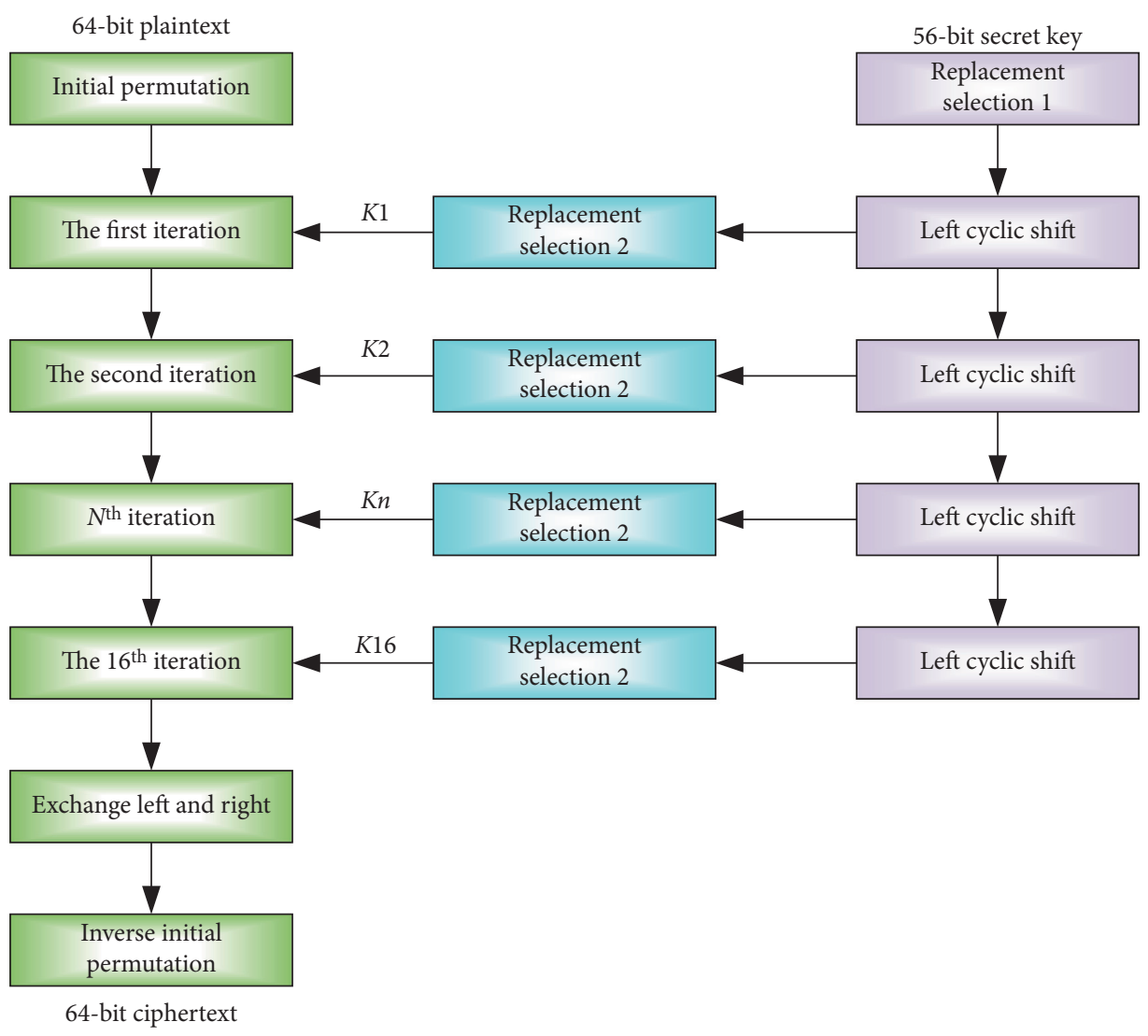

FIGURE 3: Encryption process of DES algorithm.

3.2. Implementation of Each Component of the Prototype System for Real-Time Air-to-Ground Data Communication of Health Management. The real-time communication system of air-to-ground data of health management is mainly composed of the on-board subsystem and the ground subsystem. The two subsystems are connected by the onboard terminal and ground terminal of data link.

3.2.1. On-Board Subsystem. The on-board subsystem is mainly composed of on-board data link terminal, engine simulation equipment, and flight control system.

(1) Engine simulation equipment: the STM32F405 hardware platform with a basic frequency of $168 \mathrm{MHz}$ was used for simulated operation, as shown in Figure 7, so that information such as flight status can be obtained from the on-board flight control system. Then, the engine nonlinear on-board realtime model was adopted to calculate the operating data of the simulated engine; finally, these data were sent to the data link on-board terminal, with the remote control command received from the onboard terminal of data link simultaneously.

(2) The on-board terminal of data link: the UHF communication module is shown in Figure 8, 4G communication module is shown in Figure 9, and BeiDou module is shown in Figure 10; the three data link onboard terminals are, respectively, connected to the data link comprehensive integrated unit via the serial port, the data link integrated unit is a single-chip microcomputer based on STM32L476RGT6, which mainly completes the functions of channel encoding and decoding, data packets deframing, data dispreading, modulation and demodulation, subsystem built-in test, and fault diagnosis and processing of the data link on-board terminal and then realize data communication with the engine simulation equipment and the ground terminal of data link, as shown in Figure 11, in which USART1 is connected to the $4 \mathrm{G}$ core board; USART2 is connected to the RS232 female port; UART4 is connected to the RDSS module; UART5 is connected to the UHF data transfer module; LPUART1 is connected to the engine simulation module.

(3) Flight control system: to ensure the success and safety of the flight test, this system uses the opensource flight control board Pixhawk 2.1, as shown in Figure 12. On this basis, the secondary development was carried out, and a self-defined protocol was used for communication.

3.2.2. Ground Subsystem. The ground subsystem consists of data link ground terminal, ground control center, and the simulation equipment of outfield PMA, support center, and maintenance center, as shown in Figure 13. 


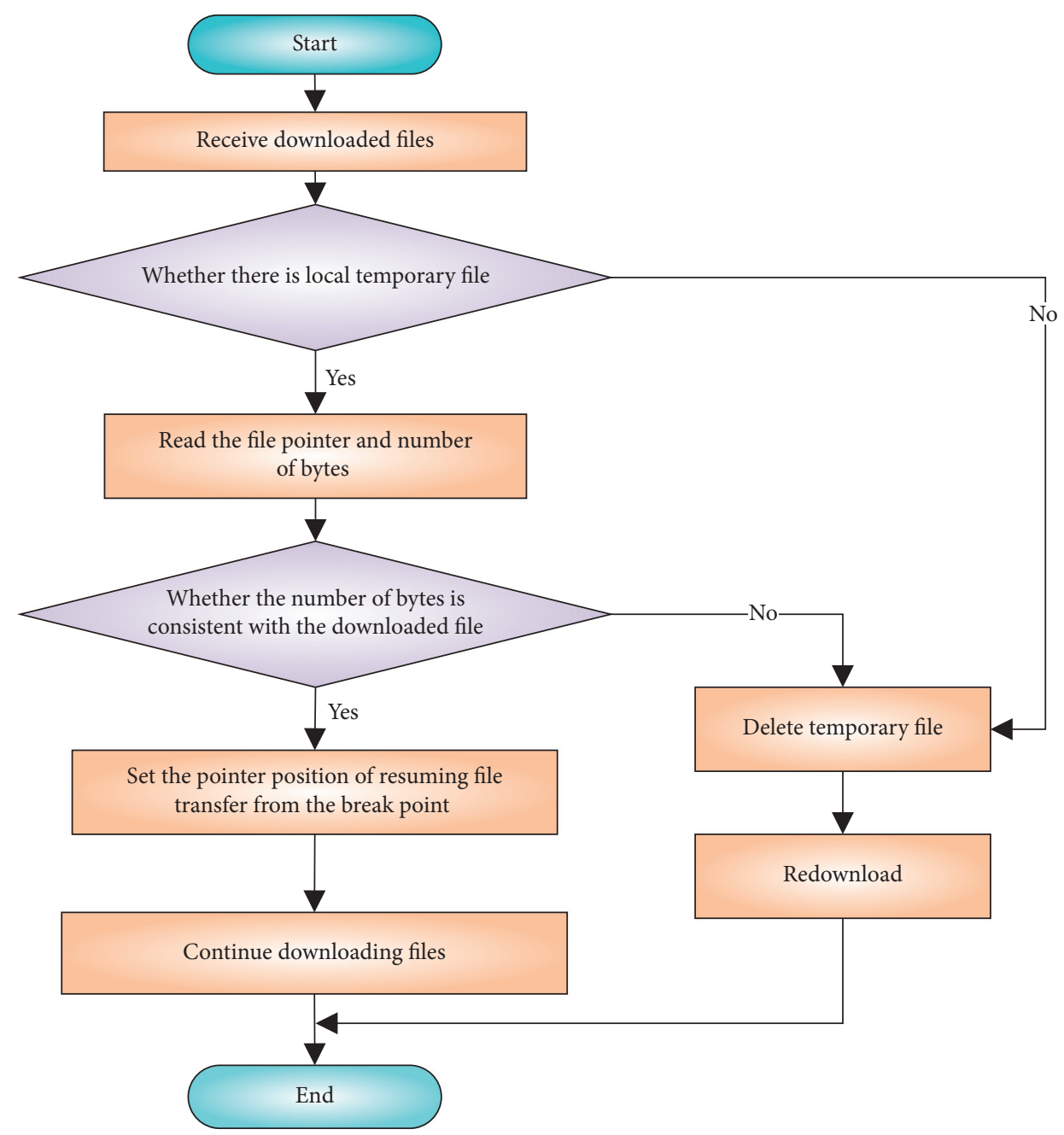

FIGURE 4: Flowchart of resuming transmission from the break point.

(1) Data link ground terminal: it mainly completes the functions of channel encoding and decoding, data packets deframing, data dispreading, modulation and demodulation, subsystem built-in test, and fault diagnosis and processing of the data link ground terminal and realizes communication with the ground control center and data link on-board terminal.

(2) Ground control center: it mainly completes the analysis of data from the ground terminal of the data link, displays the data in the form of visual charts, and sends the user's operation command to the ground terminal of data link.

(3) The simulation equipment of outfield PMA, support center, and maintenance center: it completes the communication with the ground control center and receives the data from ground control center for analysis and processing.

\section{Simulated Flight Test on Real-Time Communication and Transmission of Health Management Air-Ground Data}

4.1. Method of the Engine's Health Management Data Simulation. Due to the high cost and difficulty of collecting the health management data of real engine, interpolation was used to build a high-confidence real-time on-board engine model to simulate and calculate the working data of the engine. The parameters of each section of the current engine were obtained through the interpolation of the throttle lever command given by the flight control, and then the data of the engine were sent to the on-board terminal of data link through the serial port.

4.2. Establishment of a Flight Test Platform for Real-Time Communication and Transmission of Air-to-Ground Data. Owing to the high cost and great risk of using aircraft and 


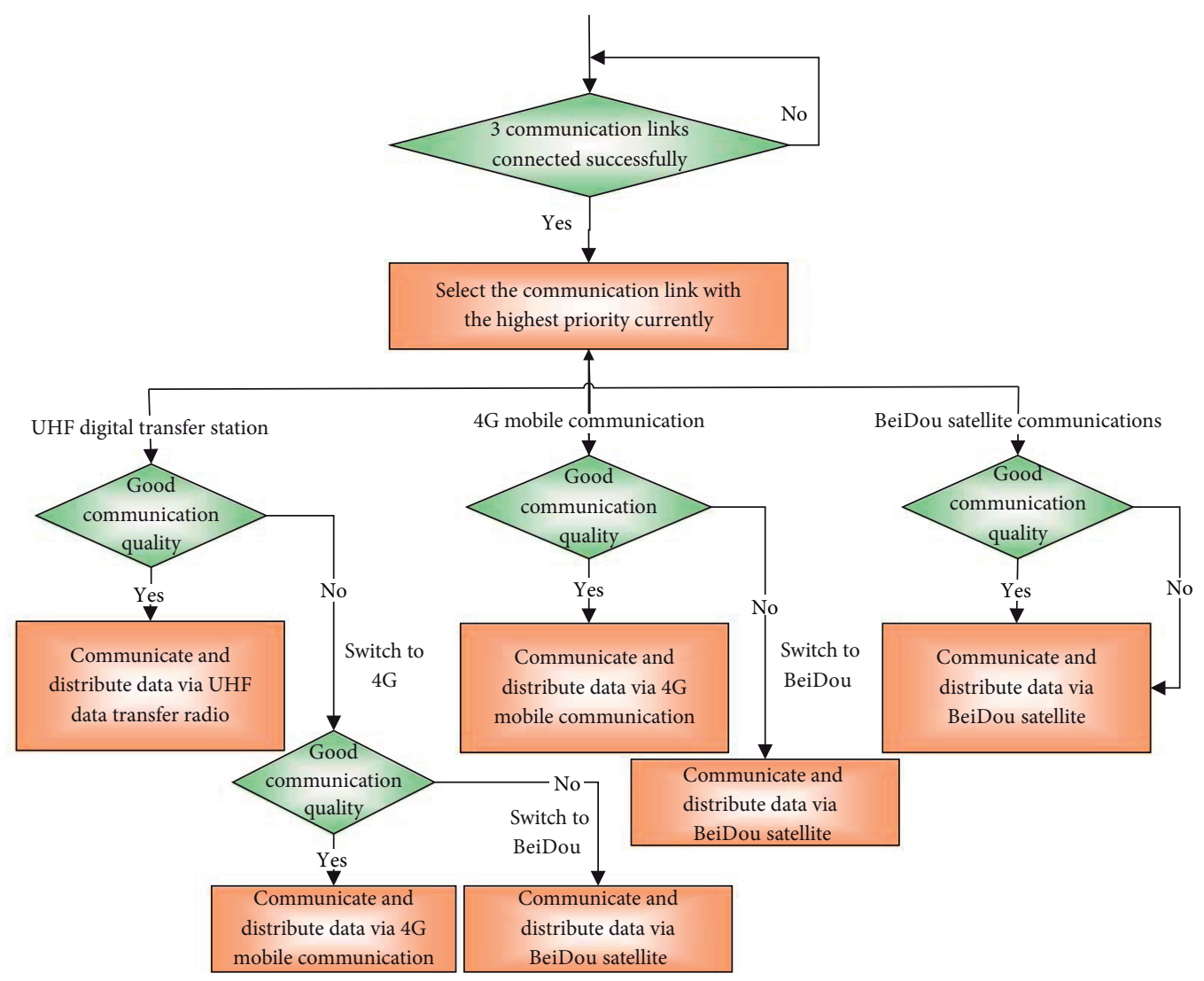

FIGURE 5: Flowchart of multilink adaptation.

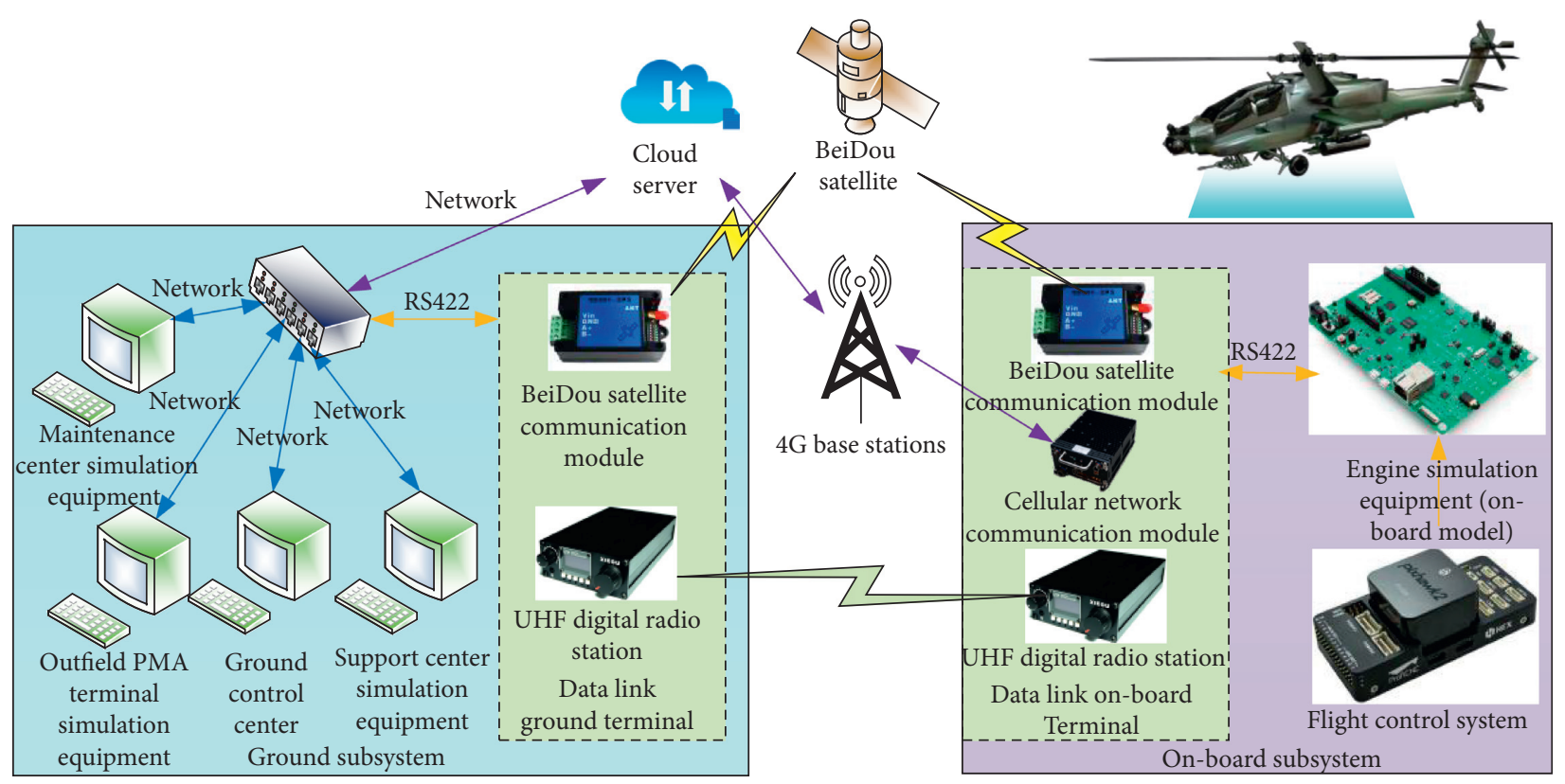

FIgURE 6: Transmission architecture of air-ground segment and ground-ground segment.

engine for data transmission simulation test, a vertical takeoff and landing UAV with fixed wings was used for test in this paper, as shown in Figure 14. Combining the advantages of rotary-wing UAV and the fixed-wing UAV, this UAV can not only achieve vertical take-off and landing but also fly horizontally with fixed wings. The functions of fixed-point take-off and landing, hovering, and high-speed cruise ensures that this UAV can operate smoothly in areas with complex terrain such as mountains, hills, and jungles and in places with dense buildings. It is characterized by fast 


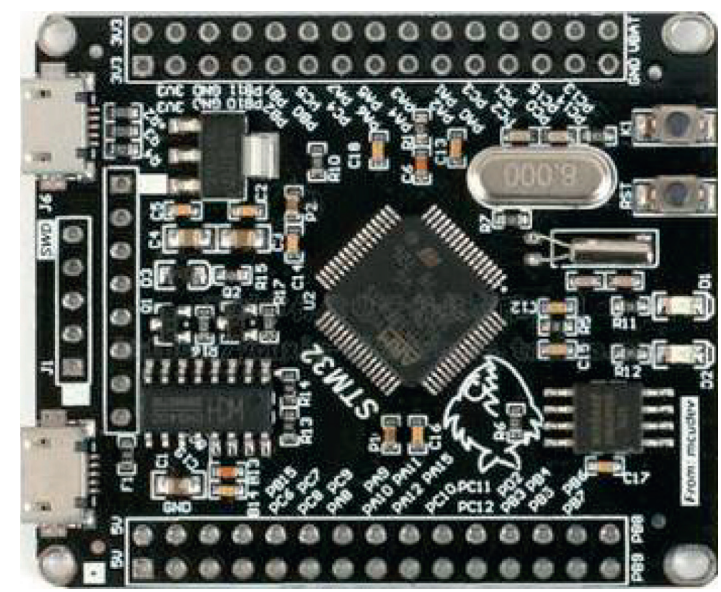

Figure 7: STM32F405 engine simulation module.

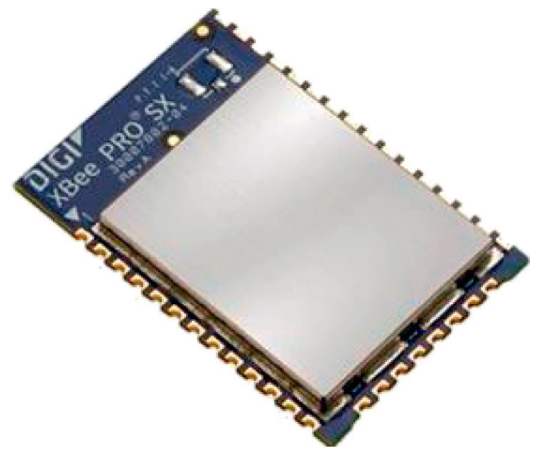

(a)

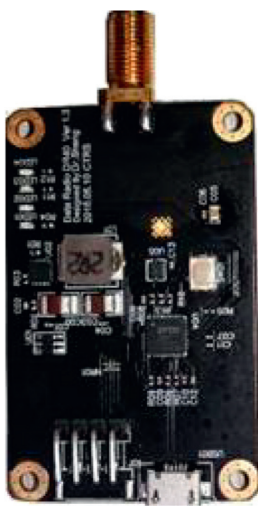

(b)

FIGURE 8: UHF communication module.

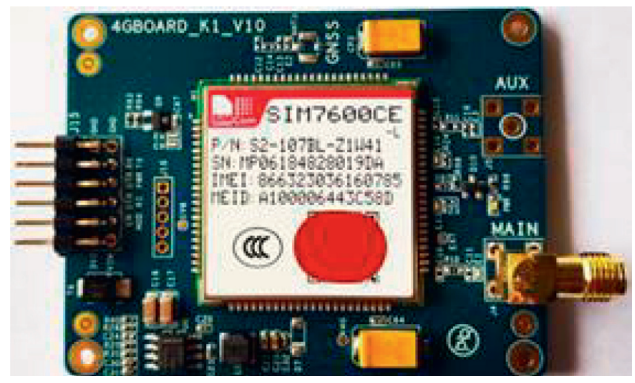

(a)

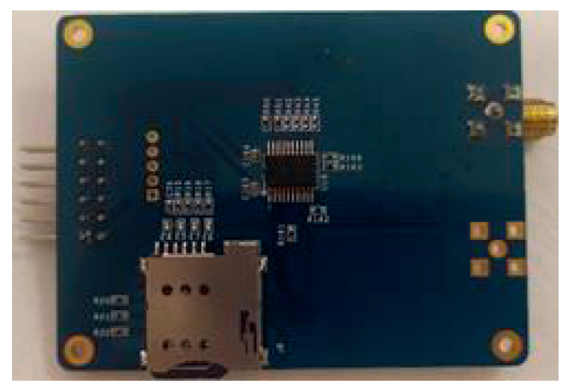

(b)

Figure 9: 4G communication module.

cruising, long flying distance, long endurance, and no terrain requirements for take-off and landing.

The schematic diagram of the test platform is displayed in Figure 15. The outfield PMA, maintenance center, support center, and ground control center are, respectively, integrated on different computers. The outfield PMA, maintenance center, and support center are connected to the ground control center through the network interface, and the ground control center is connected to the ground terminal of data link through serial port to receive the telemetry data from the ground terminal of data link for analysis and processing and forwards the received telemetry data to the outfield PMA, maintenance center, and support center for analysis and processing. In addition to the on-board flight control system, the UAV test platform is also equipped with engine simulation equipment to simulate the real-time working status of the engine and obtain data. After receiving the aircraft status 


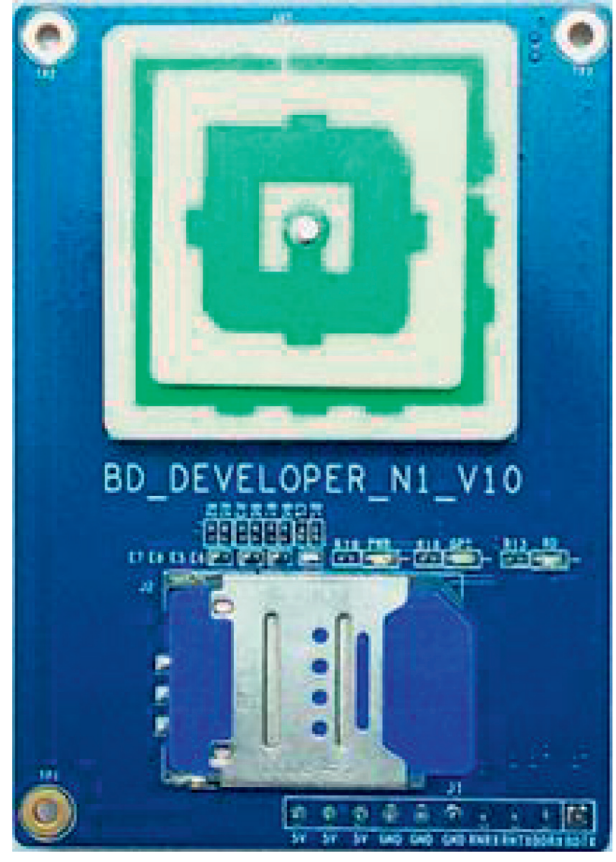

(a)

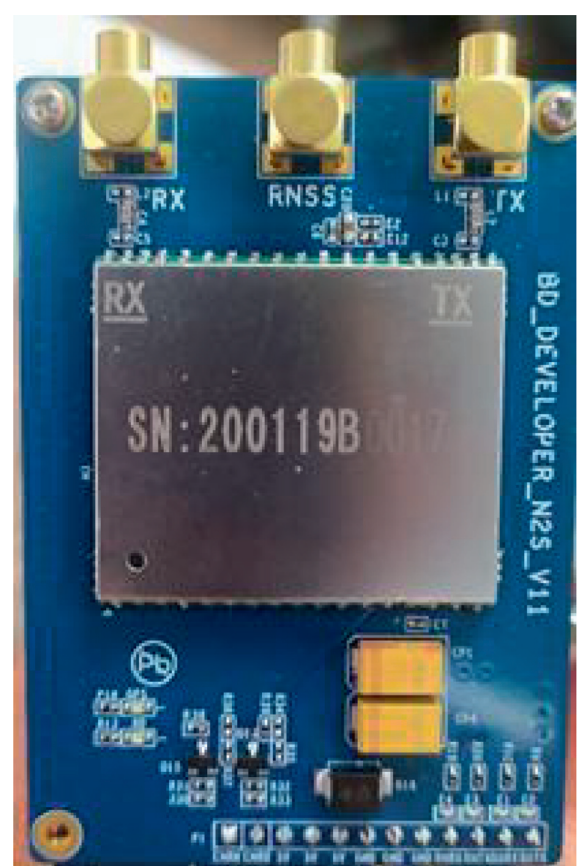

(b)

Figure 10: BeiDou communication module.

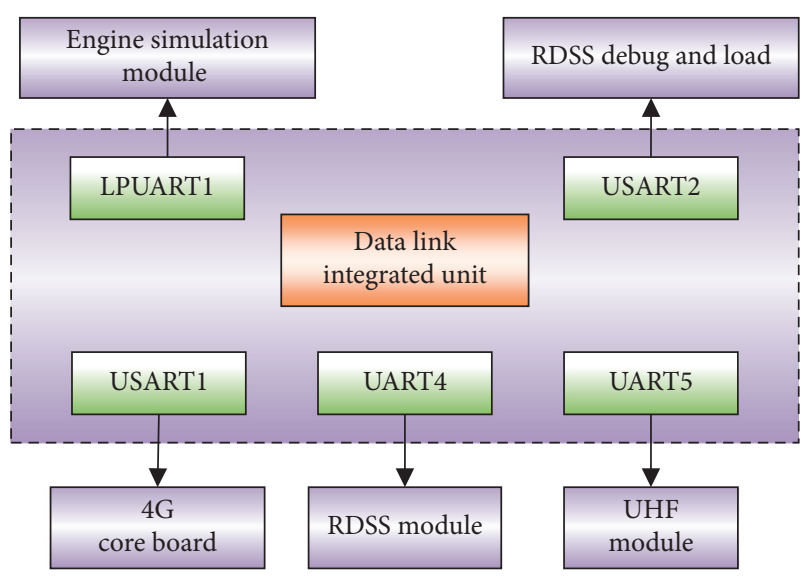

Figure 11: STM32 baseboard serial port connection.

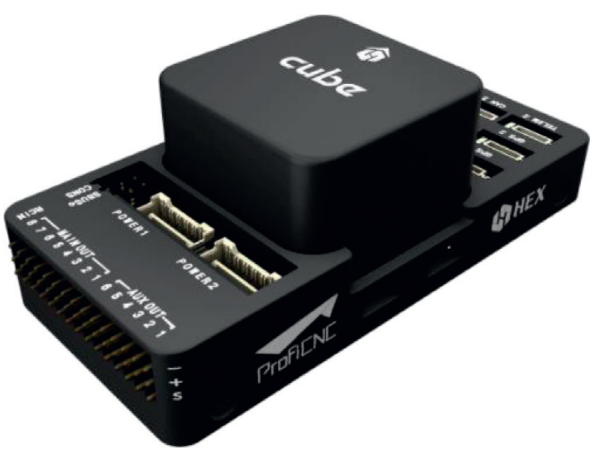

FIGURE 12: Flight control module. input from the on-board flight control system, the engine simulation equipment uses the on-board engine model to simulate changing the engine status and sends it to the on-board terminal of data link.

4.3. Simulation Test of Air-to-Ground Data Transmission of Aircraft Engine, Outfield PMA, Maintenance Center, and Support Center. To verify the technology of real-time air-toground data communication with adaptive rate of the aeroengine health management system in the whole airspace, the on-board engine model based on STM32F405 was mounted on the UAV as the engine simulation equipment for the data link communication with the ground terminal, and then the air-to-ground data transmission simulation was carried out.

According to the engine data, UAV data, and the physical characteristics of the communication links, three formats of communication protocols were defined, and three types of data messages were generated. Through the flight test data of real UAV and the engine simulation data, the communication of the air-ground links for engine's health management data was tested, involving the communication tests of the UHF data radio communication, the $4 \mathrm{G}$ cellular mobile communication link, and the BeiDou satellite communication link and the test on the adaptive switching among the three links; when the packet loss rate of the current communication link is too high or the transmission delay is too large, it can be manually or automatically switched to other communication links. In addition, with the test on the function of ground-ground segment UDP data 


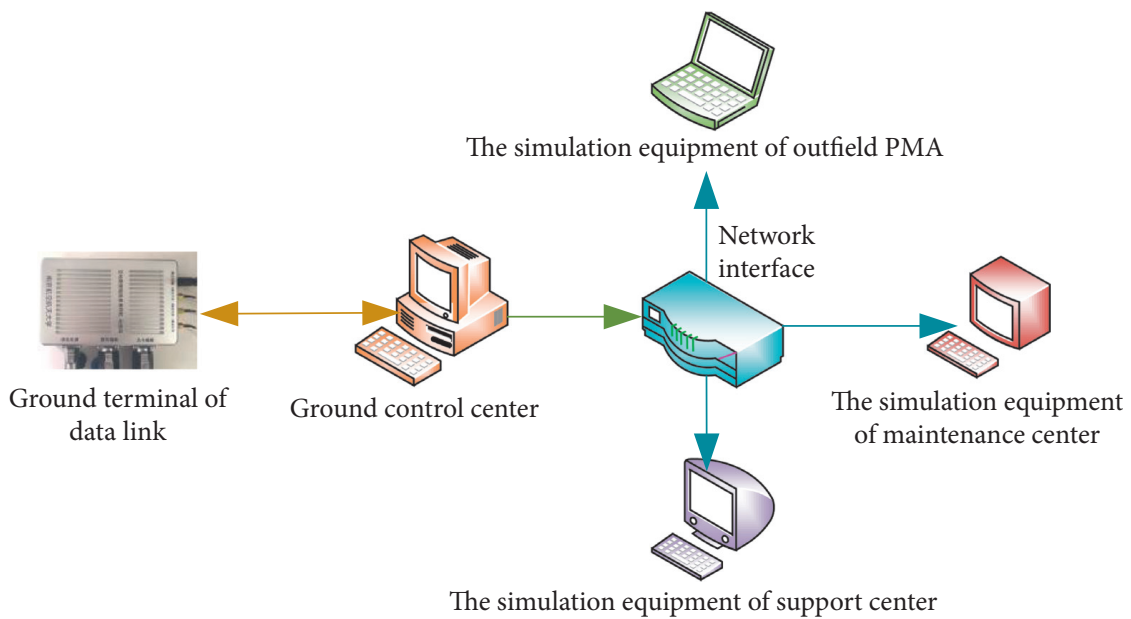

FIGURE 13: Composition of the ground subsystem.

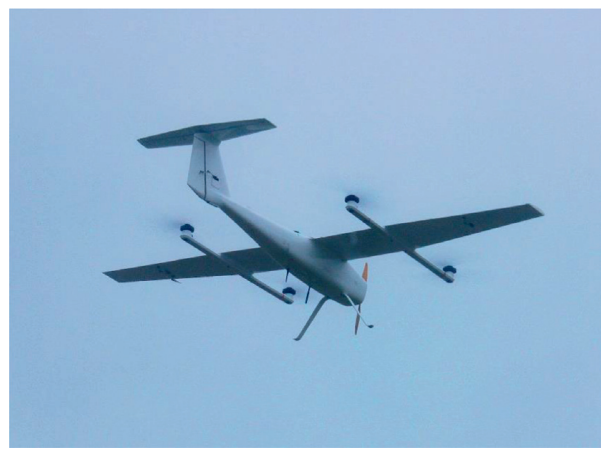

FIgURE 14: Vertical take-off and landing UAV with fixed wings.

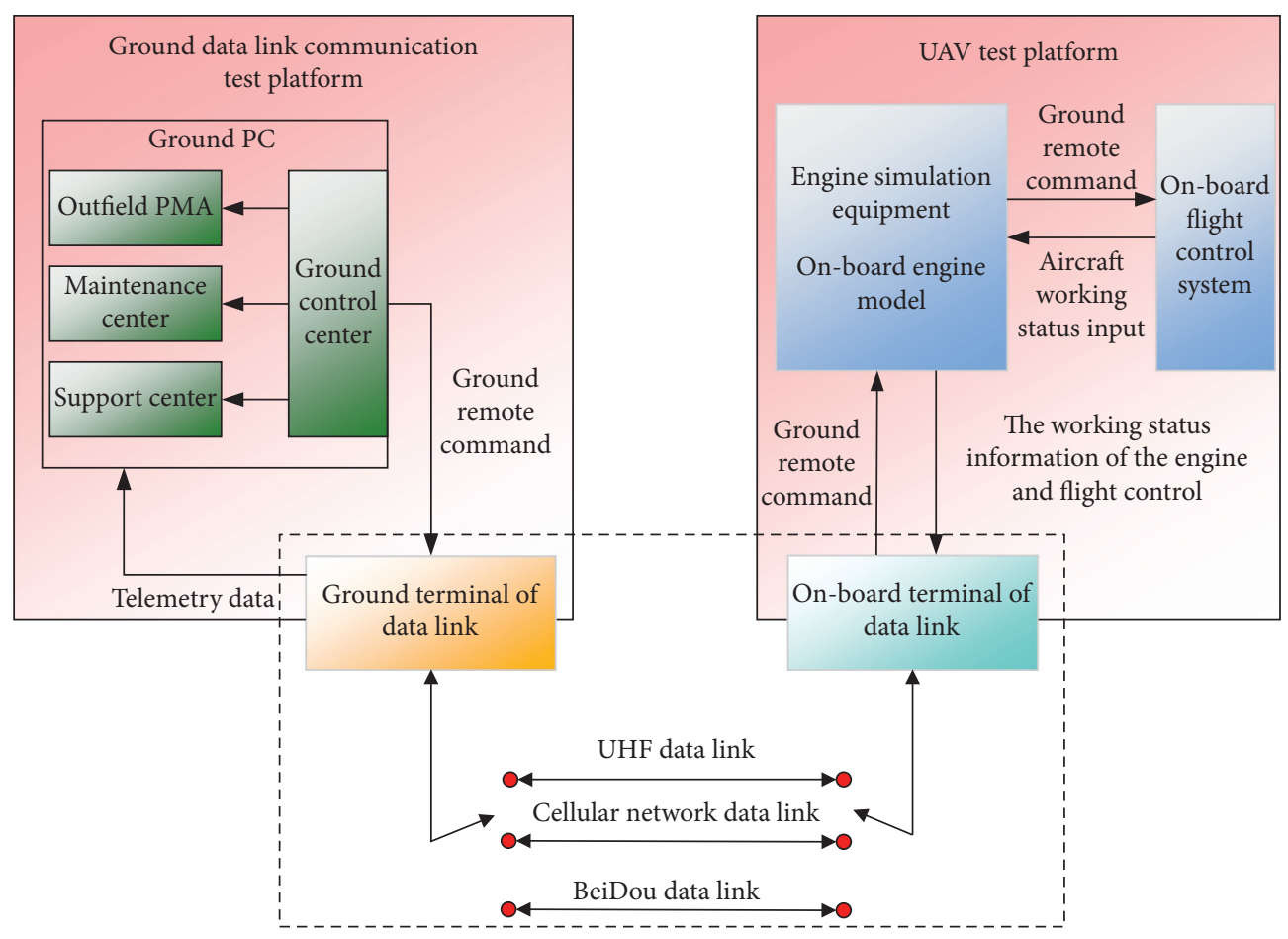

FIgURE 15: Schematic diagram of transmission simulation test platform. 


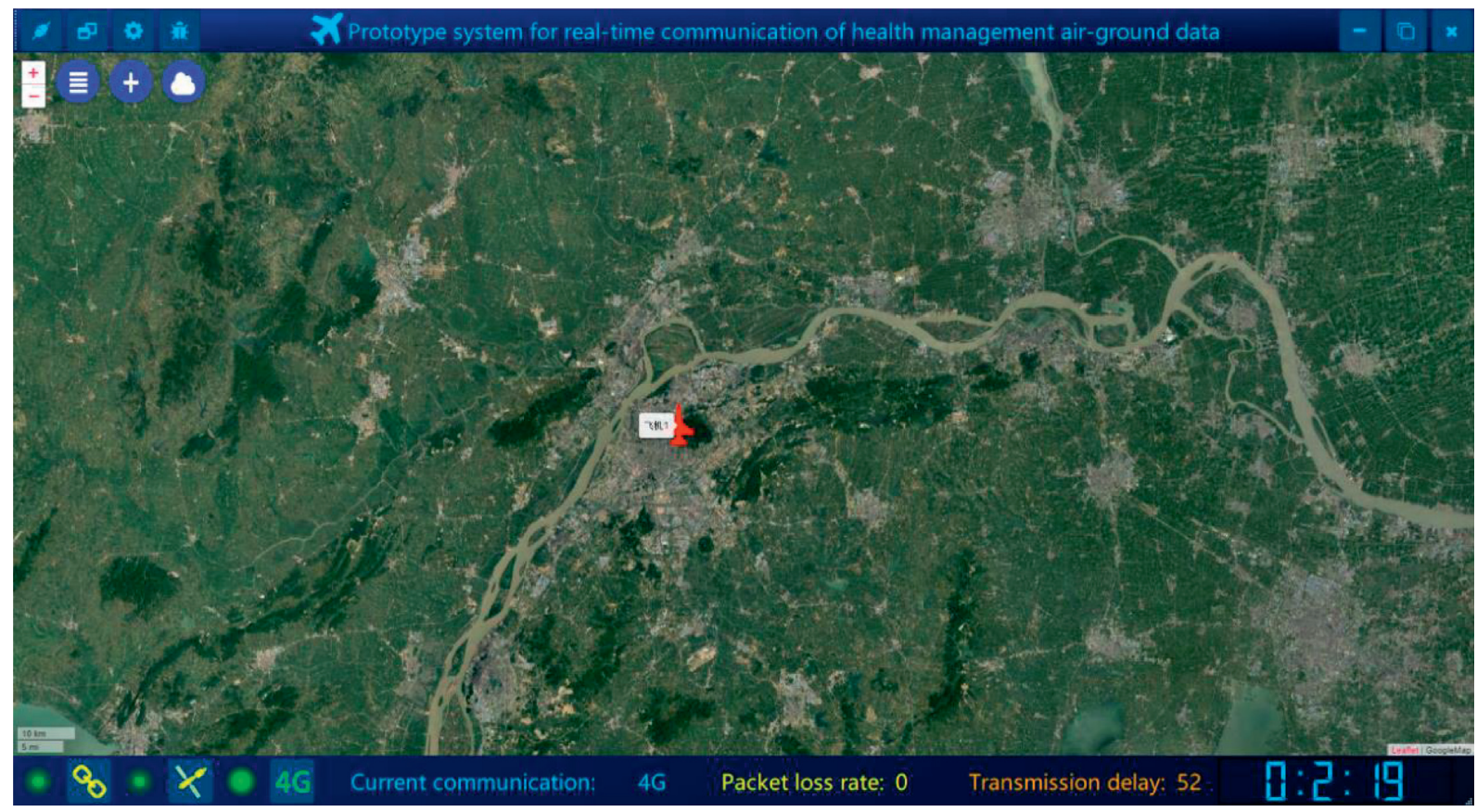

FigURE 16: 4G cellular mobile communication link air-to-ground data communication test.

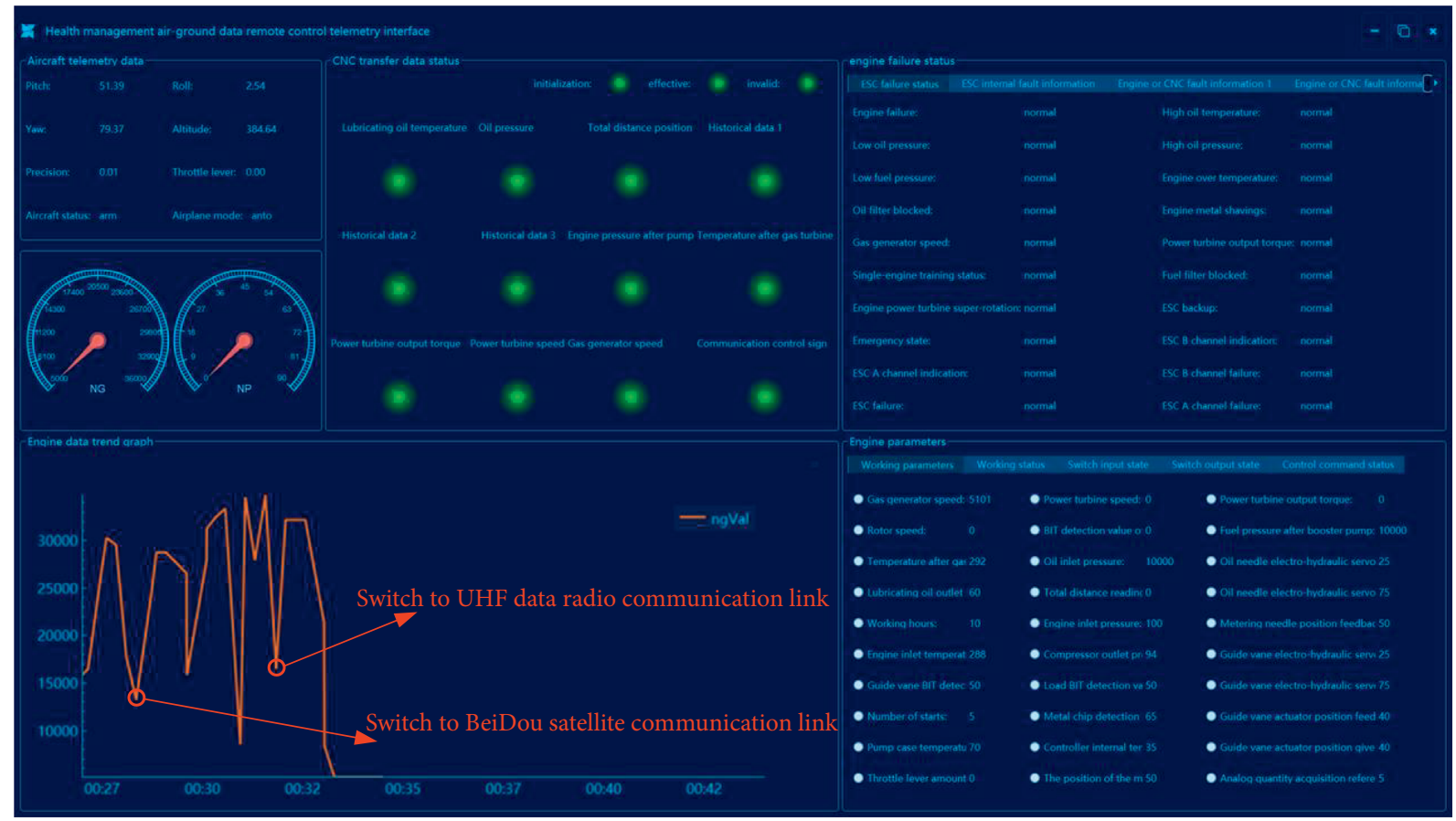

FIGURE 17: Display of the UAV/engine data switching between three communication links.

distribution, all tests passed, with the transmission delay within $100 \mathrm{~ms}$ and the packet loss rate less than $1 \%$. The entire system can accurately and effectively communicate real-time data in different flight scenarios, which greatly improves the reliability and survivability of the system. Figure 16 shows the communication test of $4 \mathrm{G}$ cellular mobile air-to-ground data. Figure 17 displays the UAV/ engine data switching from $4 \mathrm{G}$ communication link to UHF data radio communication link and then to BeiDou satellite communication link, and Figure 18 shows the communication between the ground control center and the outfield PMA, maintenance center, and support center. 


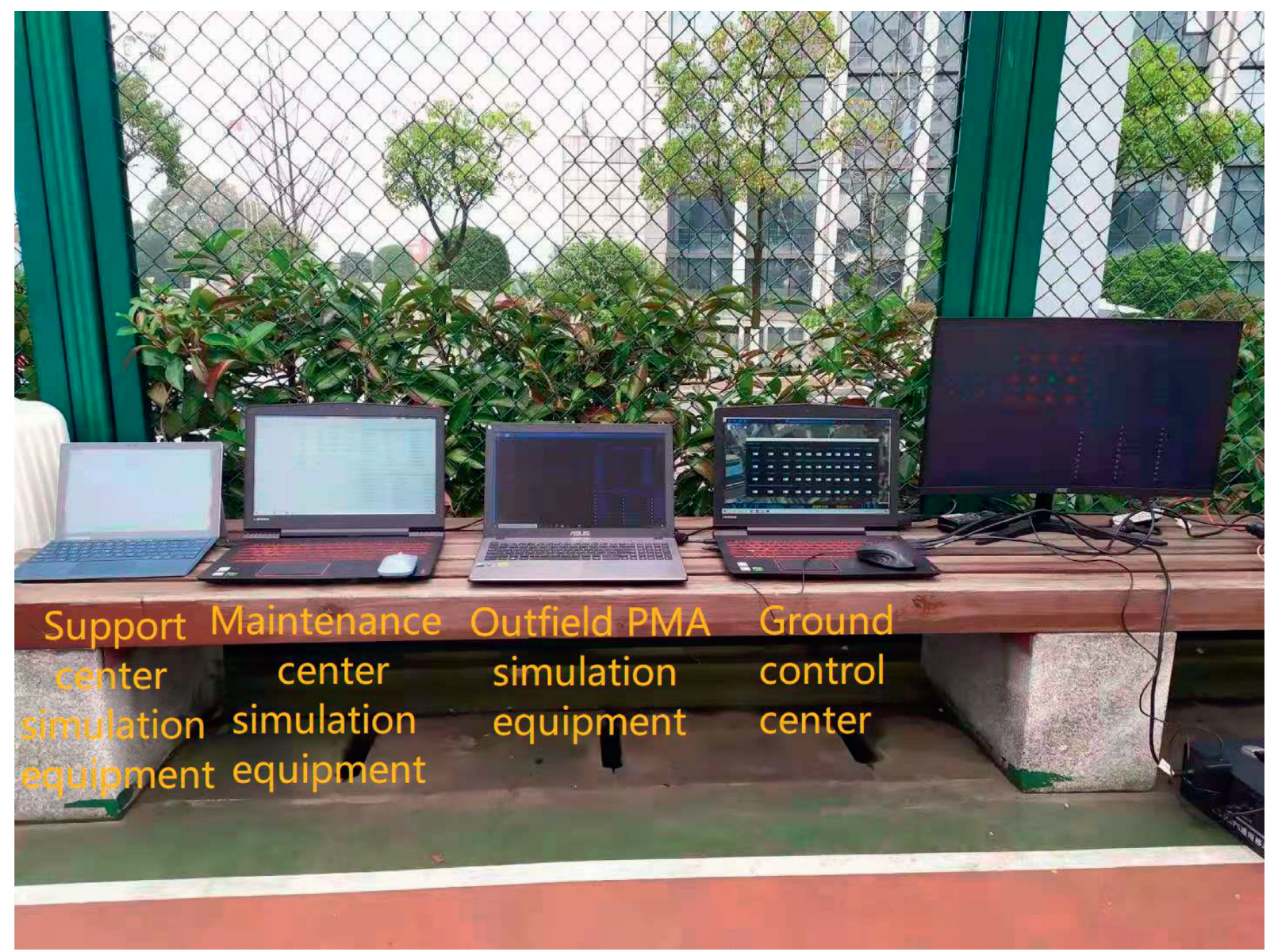

FIGURE 18: Communication between ground control center and outfield PMA, maintenance center, and support center.

\section{Problem Statement and Its Proposed Solution of Real-Time Air-to-Ground Data Communication Technology for Aeroengine Health Management System with Adaptive Rate in the Whole Airspace}

(1) At present, the communication rate of BeiDou satellite communication link is not high. It is only used in special areas without $4 \mathrm{G}$ signal coverage for emergency. In the future, BeiDou satellite communication link can be improved by using multiple BeiDou communication modules for simultaneous communication or military grade module with a communication rate of 1000 bytes per second.

(2) At present, the communication between $4 \mathrm{G}$ cellular module and server is based on TCP/IP. In the future, UDP can be used to achieve a higher communication rate.

(3) The current system only supports the resuming transfer from the break point of single file and can be improved later to realize the multiple files' resuming transfer from the break point.

\section{Conclusion}

In this paper, an aeroengine health management system was described, which realized the real-time communication of air-to-ground data in the whole airspace with adaptive rate. The system focuses on the multilink adaptive switching among UHF data transfer link, 4G cellular mobile network link, and BeiDou satellite link and also uses DES symmetric encryption algorithm and the technology of resuming transfer from the break point to ensure the security and integrity of data transmission. The simulation of air-to-ground data transmission showed that this system can realize the monitoring of aircraft and engine data and the adaptive switching among multiple links, which verified the feasibility of the system in practical application.

\section{Data Availability}

The data that support the findings of this study are available from the corresponding author upon reasonable request. 


\section{Conflicts of Interest}

The authors declare that they have no known conflicts of interest or personal relationships that could have appeared to influence the work reported in this paper.

\section{Acknowledgments}

This research work was supported by the National Natural Science Foundation of China (no. 51906103), Aviation Science Foundation of China (2018ZB52), and Aeronautics Power Foundation (6141B09050384).

\section{References}

[1] I. Y. Tumer and A. Bajwa, "A survey of aircraft engine health monitoring systems," in Proceedings of the 35th Joint Propulsion Conference and Exhibit, Los Angeles, CA, USA, June, 1999.

[2] Z. Lin and N. Zhang, "Analysis of VHF ground-to-air data link system of China's civil aviation," China Radio, vol. 2015, no. 7, pp. 45-47, 2015.

[3] C. R. Mercer, D. L. Simon, G. W. Hunter et al., Fundamental Technology Development for Gas-Turbine Engine Health Management, NASA Glenn Research Center, Cleveland, $\mathrm{OH}$, USA, 2007.

[4] M. J. Roemer, Engine Health Monitoring System for Advanced Diagnostic Monitoring for Gas Turbine Engines, Air Force Research Laboratory, Wright-Patterson Air Force Base, $\mathrm{OH}$, USA, 1998.

[5] Y. Ying, Y. Cao, S. Li, and J. Li, "Nonlinear steady-state model based gas turbine health status estimation approach with improved particle swarm optimization algorithm," Mathematical Problems in Engineering, vol. 2015, Article ID 940757, 2015.

[6] Z. Wang, J. Li, K. Fan, and S. Li, "The off-design performance simulation of marine gas turbine based on optimum scheduling of variable stator vanes," Mathematical Problems in Engineering, vol. 2017, Article ID 2671251, 2017.

[7] M. T. Yildirim and B. Kurt, "Aircraft gas turbine engine health monitoring system by real flight data," International Journal of Aerospace Engineering, vol. 2018, Article ID 9570873, 2018.

[8] L. Gou, H. Li, H. Zheng, H. Li, and X. Pei, "Aeroengine control system sensor fault diagnosis based on CWT and CNN," Mathematical Problems in Engineering, vol. 2020, Article ID 5357146, 2020.

[9] H. Zhang, G. Bai, and L. Song, "Multiobjective design optimization framework for multicomponent system with complex nonuniform loading," Mathematical Problems in Engineering, vol. 2020, Article ID 7695419, 2020.

[10] G. Mazzuto, S. Antomarioni, F. E. Ciarapica, and M. Bevilacqua, "Health indicator for predictive maintenance based on fuzzy cognitive maps, grey wolf, and K-nearest neighbors algorithms," Mathematical Problems in Engineering, vol. 2021, Article ID 8832011, 2021.

[11] Y. Yang and Z. Gao, "Power management problem for civil aircraft under more electric environment," International Journal of Aerospace Engineering, vol. 2020, Article ID 8474375, 2020.

[12] L. Rui, Y. Guo, and W. Wu, "Integrated simulation platform for the design and verification of aero-engine sensor fault diagnosis," Computer Measurement \& Control, vol. 18, no. 3, pp. 527-529, 2010.
[13] H. Yang, Aero-Engine Gas Path Fault Diagnosis Based on Intelligent Technology, Nanjing University of Aeronautics and Astronautics, Nanjing, China, 2008.

[14] X. Lv, "Discussion on the development of air-to-ground data link," Air Traffic Management, vol. 2005, no. 5, pp. 29-31, 2005.

[15] B. Sun, "Talking about the development and application of air-ground data link," Military-Civilian Dual-Use Technology and Products, vol. 2016, no. 12, p. 69, 2016.

[16] J. Huang, "Analysis of the architecture and application of civil aviation communication network," Information\& Communications, vol. 2020, no. 10, pp. 80-82, 2020.

[17] H. Wang and M. Wang, "A simulation study on V/UHF airground data link," Aircraft Design, vol. 2008, no. 1, pp. 58-63, 2008.

[18] H. Liu, "Discussion on common interference problems and countermeasures of VHF communication system (R/S200)," Digital Communication World, vol. 2020, no. 2, p. 98, 2020.

[19] Z. Huang, "Present situation and development prospect of $4 \mathrm{G}$ network technology development," Digital Communication World, vol. 2017, no. 4, pp. 46-48, 2017.

[20] J. Shen, "Overview of the development of $4 \mathrm{G}$ mobile communication technology," Wireless Internet Technology, vol. 2017, no. 7, pp. 11-13, 2017.

[21] J. Guo, "The construction, development and application of air-to-ground data link of China's civil aviation," China Civil Aviation, vol. 2006, no. 3, pp. 64-66, 2006.

[22] L. Wen, "Beidou satellite navigation system and its application in civil aviation navigation," Information Recording Materials, vol. 20, no. 7, pp. 148-149, 2019.

[23] S. Wang, R. Wang, Z. Chen et al., "Overview of aeroengine health management," Gas Turbine Test and Research, vol. 22, no. 1, pp. 51-58, 2009.

[24] The Civil Aviation Administration of China, "Roadmap for the implementation of the Beidou satellite navigation system in civil aviation of China," Civil Aviation Management, vol. 2020, no. 1, p. 76, 2020.

[25] F. Liu, D. Wang, B. Yu, and F. Yu, "Instant messaging system based on hybrid 3DES and RC4 algorithm," Computer Systems \& Applications, vol. 29, no. 8, pp. 80-89, 2020.

[26] X. Geng, "Research on file encryption based on DES algorithm," China Computer \& Communication (Theoretical Edition), vol. 32, no. 3, pp. 44-46, 2020.

[27] Y. Li, M. Cheng, X. Xu, and Y. Yang, "A Research on the continue transferring from breakpoint and multi-threading mechanism for plane media remote transmission," Journal of Beijing Institute of Graphic Communication, vol. 20, no. 6, pp. 53-56, 2012.

[28] Y. Zheng, The Broken-Point Continually Transferring and Multi-Thread Download Component Based on HTTP/FTP Protocol, University of Jinan, Jinan, China, 2012. 